\title{
$60 \mathrm{GHz}$ Path Loss Modelling Inside Ships
}

\author{
Brecht De Beelde, Emmeric Tanghe, Marwan Yusuf, David Plets, Eli De Poorter, Wout Joseph \\ Ghent University, IMEC, Ghent, Belgium, Brecht.DeBeelde@UGent.be
}

\begin{abstract}
This paper presents the results of a mmWave channel sounding campaign in a bulk carrier vessel. Using the Terragraph channel sounder, we measured path loss at $60.48 \mathrm{GHz}$ for different separations between the transmit and receiving nodes in the engine room and steering control room of the vessel. The path loss at reference distance $1.5 \mathrm{~m}$ is $\mathbf{7 4 . 6}$ $\mathrm{dB}$, which is higher than the free space path loss, whereas the path loss exponent of 1.7 is lower than in free space. The one-slope path loss model is used to estimate throughput via link budget calculations, which shows that $60 \mathrm{GHz}$ propagation realizes high data rate communication in the engine room of a vessel if the Line-of-Sight path is not obstructed. Due to the highly metallic nature of the propagation environment, reflections make communication possible in an obstructed Lineof-Sight configuration, but there is no clear distance relationship.
\end{abstract}

Index Terms-mmWave, channel characterization, vessel, path loss, modelling, channel sounding, industrial environment, ship hull, link budget.

\section{INTRODUCTION}

Wireless connectivity is omnipresent in our daily lives, from the Long-Term Evolution (LTE) technology in the smartphone to the Bluetooth Low Energy (BLE) chipset in wearables and the WiFi modem in our houses. Even though cellular and operator based technologies such as LTE, LoRA and satellite are ubiquitous, many indoor industrial areas still lack coverage as the wireless channel in industrial environments is affected by the presence of large blocking metallic constructions [1].

One such environment where metallic constructions are present is the engine room of a vessel. Currently, all data collection and equipment tracking inside the ship is a manual task by handwriting the data in a notebook. Wireless connectivity inside the ship will improve the safety and well being on board: the workers can easily access the user manuals while repairing and maintaining engine gear, the valves throughout the ship can automatically be monitored, and people and equipment can be tracked. By tracking the workers inside the engine room, an injured worker will be noticed faster. High throughput communication can enable video stream monitoring from the control room.

Path loss (PL) parameters strongly depend on the specific environment, frequency and link configuration [1]. Radio channel experiments with frequencies up to $2.6 \mathrm{GHz}$ on board of a vessel were carried out in [2] and [3], showing a path loss exponent value of less than 2 for propagation within a compartment. Such a low PL exponent is seen in several industrial environments [1]. A study on wireless sensor networks (WSNs) based on IEEE 802.15.4 on board of a vessel [4] shows very good network reliability.
For indoor environments, the $60-\mathrm{GHz}$ spectrum has been proposed to provide high-throughput communication in lineof-sight (LOS) scenarios. Channel modeling for $60-\mathrm{GHz}$ channels in indoor environments is presented in [5]-[8]. [5] investigates the spatial and temporal characteristics of multipath components (MPCs). In [6] a PL exponent value of 1.8 is found for a laboratory environment, in [8] PL exponent values below 2 are found for a data center environment.

This paper investigates the indoor radio channel at mmWave frequencies in the highly metallic environment of a vessel's engine room, in order to assess whether high data-rate wireless communication in ships is feasible. Section II provides the methodology description. The results are presented in Section III and Section IV concludes this paper.

\section{Methodology}

We investigate mmWave propagation in the engine room of a vessel via an empirical approach by using a $60 \mathrm{GHz}$ channel sounder consisting of a customized pair of Terragraph (TG) nodes [9]. This channel sounder uses a grid of 36x8 vertically polarized transmit/receive antennas with independently controlled phase shifters and measures directional path loss and channel impulse response. The spacing of the antenna elements is $0.55 \lambda$. By adjusting the phase of the antenna elements, the sounder is capable of electrically scanning in the azimuth plane in a range from $-45^{\circ}$ to $45^{\circ}$ with a beam width of $2.8^{\circ}$.

\section{A. Channel sounder}

The channel sounder successively transmits IEEE802.11ad frames and performs a power measurement of the received signal for every azimuth angle of the transmitter (TX) and receiver $(\mathrm{RX})$. The measurement data is interpolated with calibration files in order to determine path loss for every angle combination in the azimuth plane of the TX and RX. As the inter-element spacing is larger than $\lambda / 2$ and as there is only a phase adjustment for the antenna elements without gain tapering, sidelobes are present.

\section{B. Measurement environment}

We performed 76 Line-of-Sight (LOS) measurements at different locations inside the hull of a $200 \mathrm{~m}$ long bulk carrier vessel. These locations are displayed in Fig. 1a. For every location, the location of the TX node is fixed and measurements are performed with the RX node at different distances (moves along the line depicted in Fig. 1a). Combining the measurements from all locations, we get PL data for distances ranging from $1.5 \mathrm{~m}$ to $14.25 \mathrm{~m}$ with a spacing of $25 \mathrm{~cm}$. 


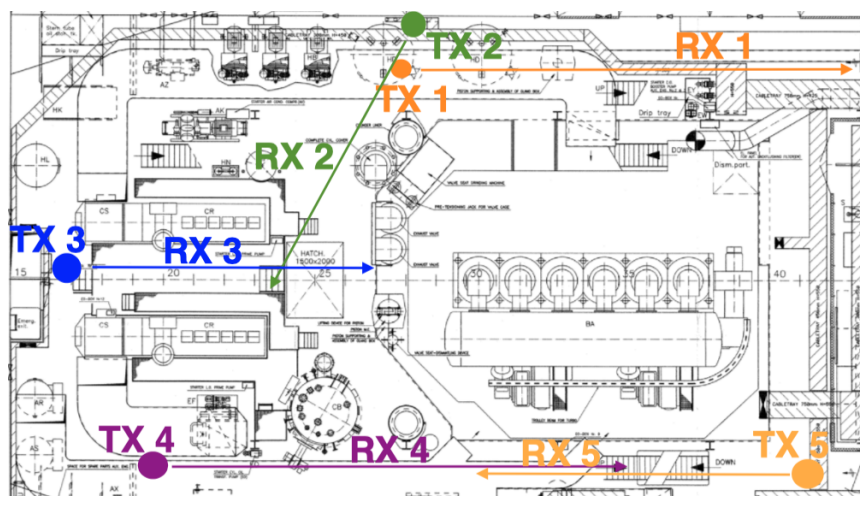

(a) Floorplan of the engine room with TX locations and RX tracks of LOS measurements

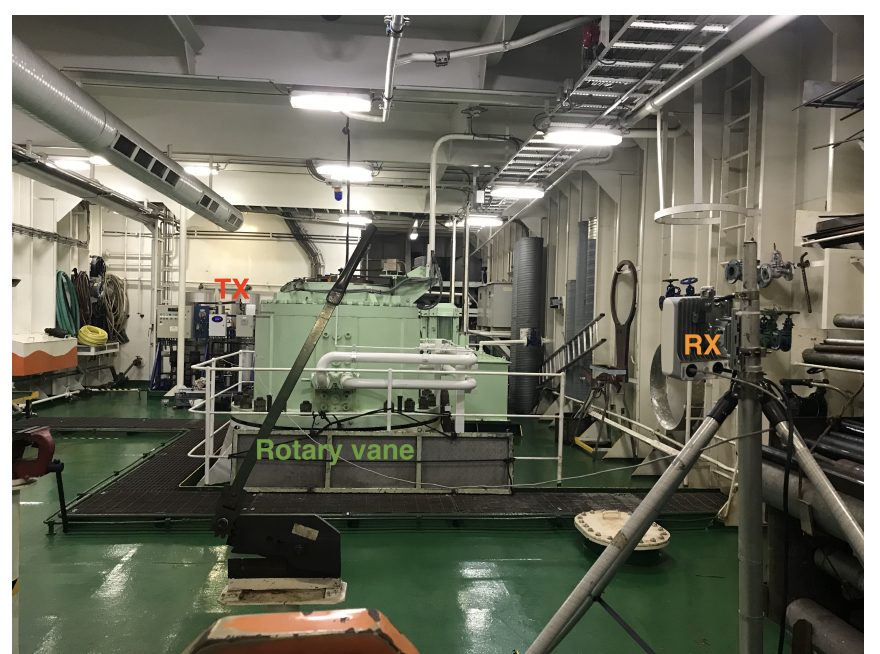

(b) Picture of steering room where the rotary vane is obstructing LOS path between TX and RX

Fig. 1: Overview measurement locations in the vessel

Next to the LOS measurements in the engine room, we also performed 6 obstructed Line-of-Sight (OBS) measurements in the steering control room, with the rotary vane obstructing the Line-of-Sight path between the two nodes. This measurement setup is shown in Fig. 1b. The distance between TX and $\mathrm{RX}$ ranges from 8 to $12 \mathrm{~m}$. During the measurements, the auxiliary engine responsible for the electricity generation on board, next to the receiver at location 3 was running, causing vibrations of the equipment and environment. The environment is considered stationary as there were no people moving around during the measurements.

\section{Signal attenuation}

The channel sounder measurement returns received power (transformed to a path loss value) for every TX-RX beam combination. For every measurement, we select the TX-RX beam combination corresponding to the minimum PL. The PL values are fitted to the one-slope model presented in (1) with regression parameters $P L_{0}$ (in $\mathrm{dB}$ ), the PL at a reference distance $d_{0}$ of $1.5 \mathrm{~m}$ (as in [8]), and PL exponent $n(-) . \chi$ is zero-mean normally distributed shadow fading term in $\mathrm{dB}$.

$$
P L(d)=P L_{0}+10 n \log _{10}\left(d / d_{0}\right)+\chi
$$

We investigate the spatial characteristics in the engine room by visualizing the PL for every TX-RX beam combination.

\section{Delay spread}

Root-mean-squared (RMS) delay spread is obtained from channel impulse response (CIR) estimation based on the training sequence of the IEEE802.11ad preamble. The TG channel sounder performs a 256-tap complex CIR estimation at a sampling rate of $3.52 \mathrm{GHz}$. We obtain the RMS delay spread for all TX-RX combinations at select distances and compare the RMS delay spread of the LOS measurements with the OBS measurements.

\section{E. Link budget calculation}

The link budget equation presented in (2) is used to estimate throughput as a function of distance. It contains the transmit power $P_{T}$ (in $\left.\mathrm{dBm}\right)$, transmit/receiver gains $G_{T}, G_{R}$ (in $\mathrm{dBi}$ ) and losses $L_{T}, L_{R}$ (in $\mathrm{dB}$ ), as well as the path loss $P L$ (in $\mathrm{dB})$ and the receiver sensitivity $P_{R S}$ (in $\mathrm{dBm}$ ).

$$
P_{R}=P_{T}+G_{T}+G_{R}-L_{T}-L_{R}-P L-P_{R S}
$$

Table I lists receiver sensitivities for different modulation and coding schemes (MCS) for IEEE802.11ad using a single carrier PHY. These values are taken from [11].

\section{Results}

\section{A. Angular profile of measured $P L$}

Figures $2 \mathrm{a}$ and $2 \mathrm{~b}$ show the measured PL as a function of the TX and RX azimuth angles for the LOS measurements at location 4 in the engine control room for distances of $2 \mathrm{~m}$ and $8.5 \mathrm{~m}$ between TX and RX nodes. The cross pattern is caused by the sidelobes of the RX aligning with the main lobe of the transmitter and vice versa. From the LOS measurements, we see that more reflections are present in the angular profile for higher distances between the two nodes.

Figures $2 \mathrm{c}$ and $2 \mathrm{~d}$ display the angular profile of the OBS measurements in the steering control room for two different distances. From these angular profiles, we see that a small change in distance significantly changes the multipath components between a transmitter and receiver in an OBS scenario. The attenuation of the LOS component for a distance of $10 \mathrm{~m}$ is $113 \mathrm{~dB}$, which is $25 \mathrm{~dB}$ higher than the free space PL for the same distance. The main reflected path (with TX and RX beams of $+-34^{\circ}$ ) has a slightly lower attenuation of $109.6 \mathrm{~dB}$.

\section{B. Path loss}

Figure 3 presents the minimum measured PL values together with a one slope model of the combined data set. The fitted path loss $P L_{0}$ at reference distance $d_{0}=1.5 \mathrm{~m}$ is $74.6 \mathrm{~dB}$, which is higher than the free space PL of $71.6 \mathrm{~dB}$ at the same reference distance. However, the fitted PL exponent $n$ of 1.68 is lower than the free space PL exponent of 2 . 
TABLE I: Receiver sensitivity $\left(P_{R S}\right)$ and Data Rate (DR) vs. modulation and coding scheme (MCS)

\begin{tabular}{|l|c|c|c|c|c|c|c|c|c|c|c|c|c|}
\hline MCS & 0 & 1 & 2 & 3 & 4 & 5 & 6 & 7 & 8 & 9 & 10 & 11 & 12 \\
\hline$P_{R S}[\mathbf{d B m}]$ & -78 & -68 & -66 & -64 & -64 & -62 & -63 & -62 & -61 & -59 & -55 & -54 & -53 \\
\hline DR [Mbps] & 27.5 & 385 & 770 & 962.5 & 1155 & 1251 & 1540 & 1925 & 2310 & 2502 & 3080 & 3850 & 4620 \\
\hline
\end{tabular}

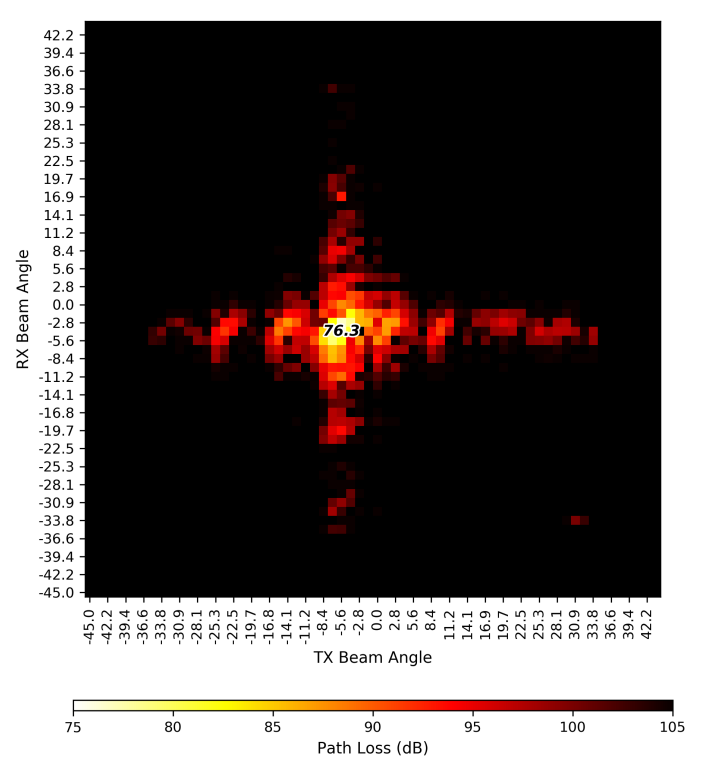

(a) LOS loc. 4, distance $2 \mathrm{~m}$

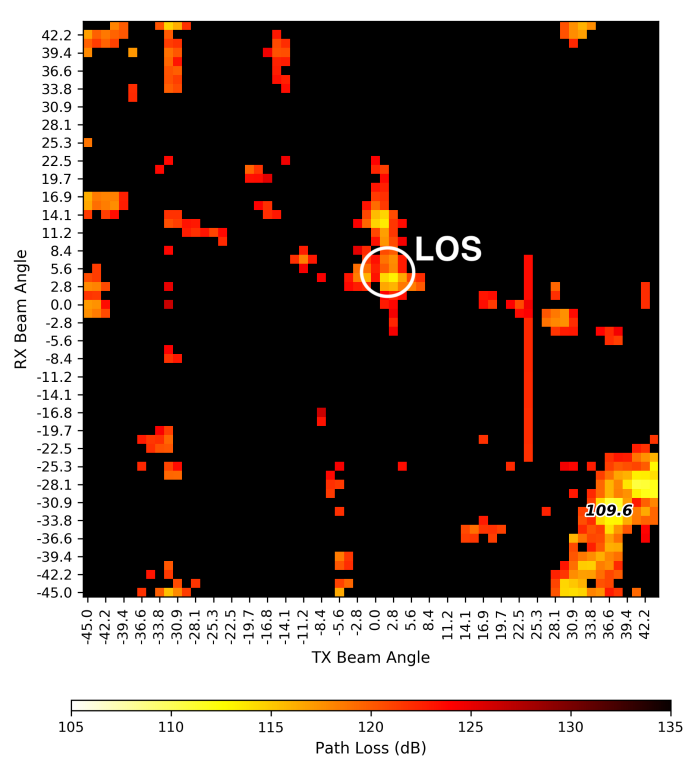

(c) OBS, distance $10 \mathrm{~m}$

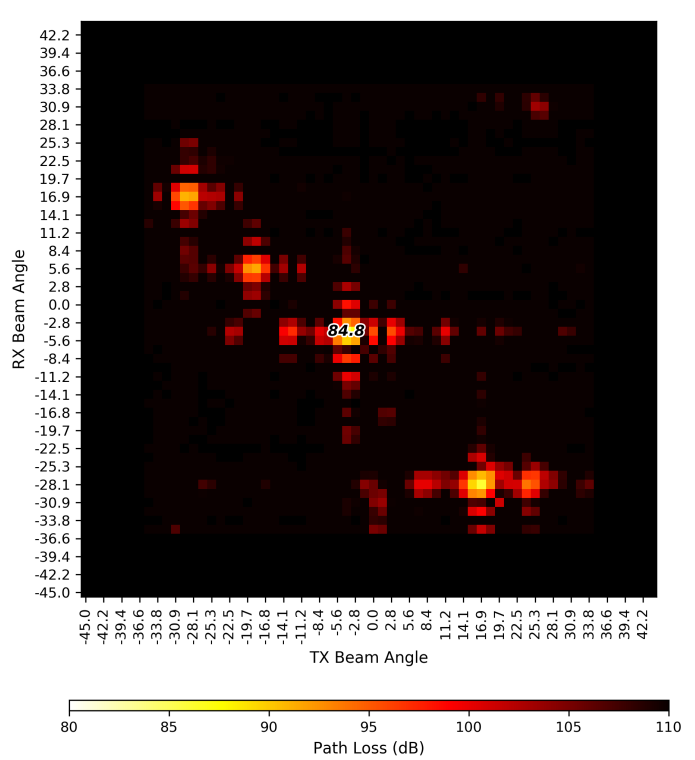

(b) LOS loc. 4, distance $8.5 \mathrm{~m}$

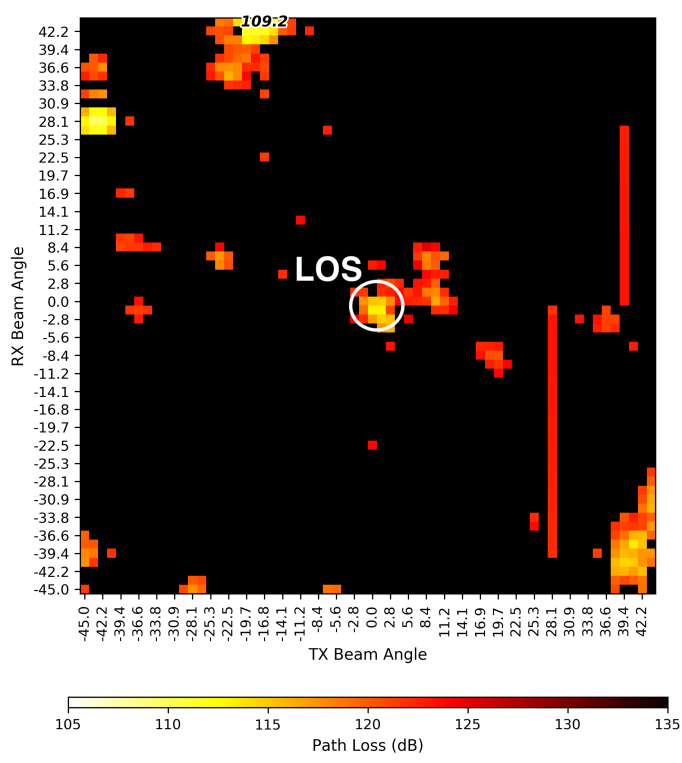

(d) OBS, distance $12 \mathrm{~m}$

Fig. 2: Measured PL as a function of TX-RX beam configuration 


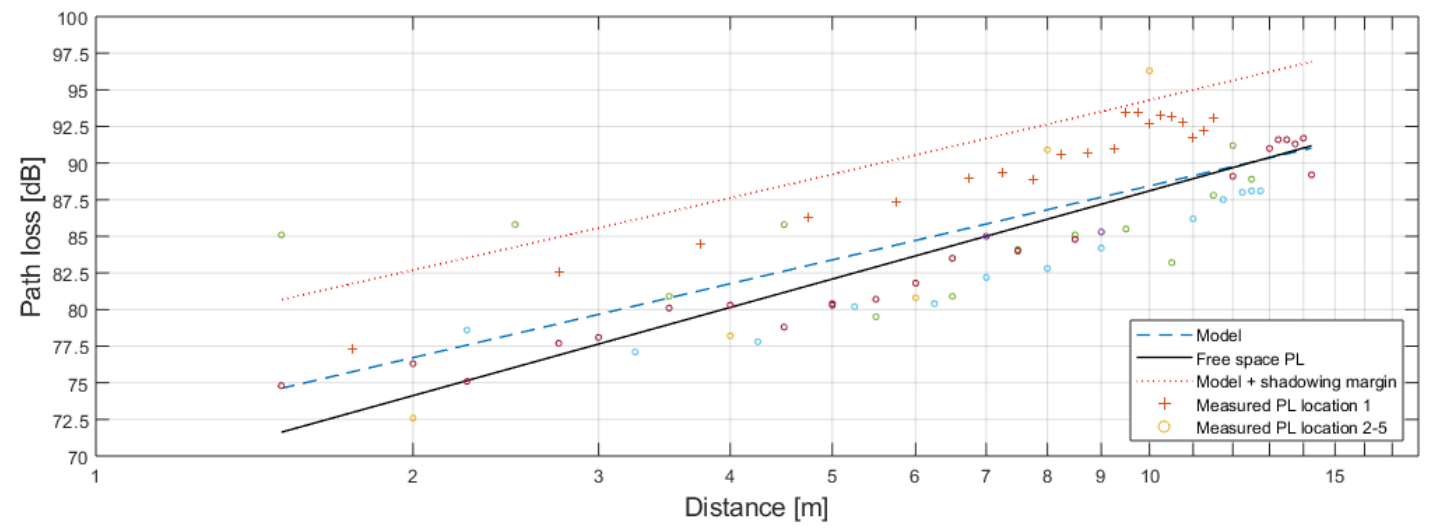

Fig. 3: Line-of-Sight PL model for the engine room of a vessel

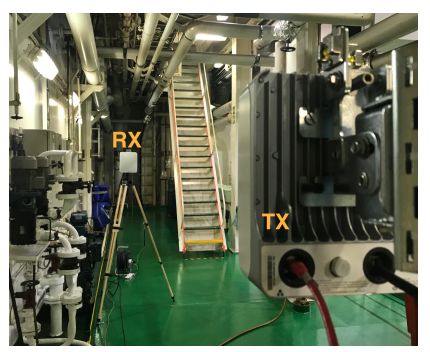

(a) Loc. 1, higher measured PL

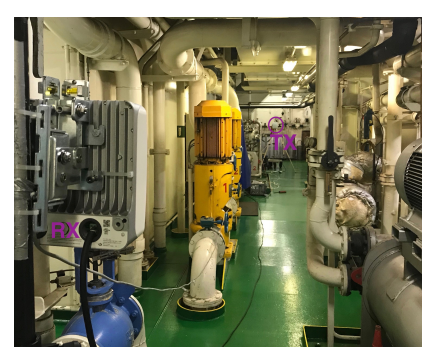

(b) Loc. 4, lower measured PL

Fig. 4: Measurement locations 1 and 4 in engine room

The root mean squared error (RMSE) between the model and measurement data is $3.48 \mathrm{~dB}$, which results in an added p95 shadow margin of $5.8 \mathrm{~dB}$ shown as the red dotted line in Fig. 3. This figure shows that for location 1 , next to the wall furthest away from the main engine and presented by plus signs, the measured $\mathrm{PL}$ is around $5 \mathrm{~dB}$ higher than free space PL, whereas the measured PL for locations 2 to 5 (presented by circles) is close to or lower than free space PL. This could be explained by room geometry, as location 1 was more open compared to the other locations, as can be seen from Fig. 4 .

In Section III-A, we have seen that there is a wider angular spread in an obstructed Line-of-Sight scenario. The reflected components have lower attenuation than the LOS path, hence the PL depends more on the room geometry and reflectors and less on the distance between the two nodes. The PL of the obstructed LOS path is about $25 \mathrm{~dB}$ higher than the minimum PL of the LOS measurements.

\section{RMS Delay spread}

In a highly metallic environment, wireless communication is not only challenged by signal blockage, but also by RMS delay spread. The RMS delay spread is obtained from the CIR channel estimation. As an example, the CIR of the LOS measurement at location 4 is shown in Fig. 5 when the distance between the nodes was $4.75 \mathrm{~m}$. For all TXRX combinations of the LOS measurements, median RMS

delay spread values range from 0.8 to $6.3 \mathrm{~ns}$, but the RMS delay spread corresponding to the LOS beam (i.e. TX and RX angle $0^{\circ}$ ) is smaller than $0.5 \mathrm{~ns}$ for all distances. For the OBS measurements, the RMS delay spread of the beam combination with minimum PL ranges from 1.5 ns to $2.6 \mathrm{~ns}$.

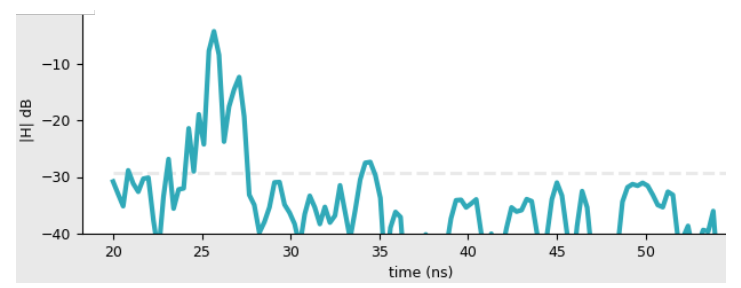

Fig. 5: Channel Impulse Response at LOS location 4 with distance $4.75 \mathrm{~m}$ between the two nodes, using a TX-RX beam configuration of -1.4 degrees for the TX node and -4.2 degrees for the RX node.

\section{Link budget}

Using the PL model of Section III-B, IEEE802.11ad throughput is calculated as a function of distance using the link budget of Eq. (2). Transmit power is assumed to be $13 \mathrm{dBm}$, the antenna gains are considered to be $8 \mathrm{dBi}$, insertion loss $L_{T}$ is $1 \mathrm{~dB}$ and $L_{R}$ is assumed to be zero. A shadow margin of $5.8 \mathrm{~dB}$ is added for a $95 \%$ quantile. Figure 6 shows the throughput based on the receiver sensitivity values from Table I.

\section{CONCLUSION}

In this paper, the wireless channel at $60 \mathrm{GHz}$ in ships is modelled. The reference path loss $74.6 \mathrm{~dB}$ (at distance $1.5 \mathrm{~m}$ ) is higher than the free space path loss. The path loss exponent of 1.7 is lower than in free space due to the metallic environment. In an obstructed Line-of-Sight scenario, propagation via a reflected path is a few $\mathrm{dB}$ better than the obstructed LOS path, but the obstructed LOS component is up to $25 \mathrm{~dB}$ more attenuated than a non-obstructed LOS path. This makes the higher MCS codes unusable for OBS. 


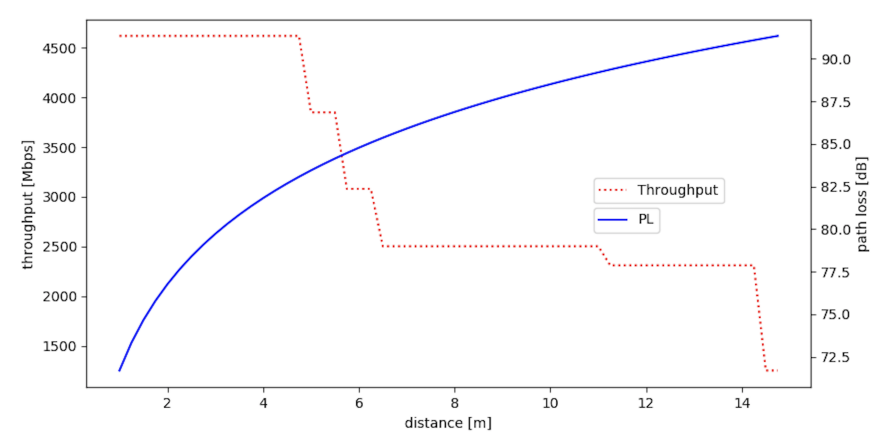

Fig. 6: Throughput and path loss as a function of distance

\section{ACKNOWLEDGMENT}

This work was executed within the Internet-of-Shipping (IoS) research project. IoS is co-financed by imec and received support from Flanders Innovation \& Entrepreneurship. The authors would like to thank Ivan Renette from Exmar Shipmanagement to facilitate the measurement campaign.

The channel sounder used for the experiments has been granted as part of the Channel Sounder Program of the mmWave Networks Project Group of Telecom Infra Project (TIP) [12].

\section{REFERENCES}

[1] M. Cheffena, Propagation Channel Characteristics of Industrial Wireless Sensor Networks [Wireless Corner], IEEE Antennas and Propagation Magazine, vol. 58, no. 1, pp. 66-73, Feb. 2016.
[2] E. Balboni, J. Ford, R. Tingley, K. Toomey, and J. Vytal, An empirical study of radio propagation aboard naval vessels, IEEE-APS Conference on Antennas and Propagation for Wireless Communications, Waltham, MA, USA, Nov. 2000, pp. 157-160.

[3] D. Estes, T.B. Welch, A.A. Sarkady, H. Whitesel, Shipboard radio frequency propagation measurements for wireless networks, Military Communication Conerence, Washington DC, USA, 2001, pp. 247-251.

[4] H. Kdouh, G. Zaharia, C. Brousseau, G. Grunfelder, H. Farhat and G. El Zein, Wireless Sensor Network on board vessels 2012 19th International Conference on Telecommunications (ICT), Jounieh, 2012, pp. $1-6$.

[5] Hao Xu, V. Kukshya and T. S. Rappaport, Spatial and temporal characteristics of 60-GHz indoor channels, IEEE Journal on Selected Areas in Communications, vol. 20, no. 3, pp. 620-630, April 2002.

[6] Moraitis N, Constantinou P, Indoor channel measurements and characterization at $60 \mathrm{GHz}$ for wireless local area network applications, IEEE Transactions on Antennas and Propagation, vol. 52, no. 12, pp. 3180-3189, 2004 Dec.

[7] K. Haneda, J. Järveläinen, A. Karttunen, M. Kyrö and J. Putkonen, A Statistical Spatio-Temporal Radio Channel Model for Large Indoor Environments at 60 and $70 \mathrm{GHz}$, IEEE Transactions on Antennas and Propagation, vol. 63, no. 6, pp. 2694-2704, 2015.

[8] M. Z. Zaaimia, R. Touhami, L. Talbi, M. Nedil and M. C. E. Yagoub, 60-GHz Statistical Channel Characterization for Wireless Data Centers, IEEE Antennas and Wireless Propagation Letters, vol. 15, pp. 976-979, 2016

[9] Facebook Connectivity, Terragraph initiative, https://terragraph.com

[10] H. Xu, T. S. Rappaport, R. J. Boyle, J. Schaffner, Measurements and modeling for 38-GHz point-to-multipoint radiowave propagation, IEEE J. Select. Areas Commun., vol. 18, pp. 310-321, Mar. 2000.

[11] IEEE Std 802.11adTM-2012, Part 11: Wireless LAN Medium Access Control (MAC) and Physical Layer (PHY) Specifications Amendment 3:, Enhancements for Very High Throughput in the $60 \mathrm{GHz}$ Band

[12] Telecom Infra Project, Millimeter Wave (mmWave) Networks Project Group, Channel Modelling track, https://mmwave.telecominfraproject.com 